\title{
Frequency of Patients Passing an Impacted Esophageal Meat Bolus by Conservative Method Alone
}

\section{Ghulam Murtaza, ${ }^{1}$ Sarwat Hassan Syed, ${ }^{2}$ Damish Arsalan, ${ }^{3}$ Mohammad Qamar Nasir, ${ }^{4}$ Muhammad Awais Amin, Amir Ayub Awan ${ }^{6}$}

\begin{abstract}
Objectives: To determine the frequency of patients passing the impacted esophageal meat bolus by conservative management

Methods: After obtaining permission from ethical committee of Hospital,a total of 62 patients meeting the study criteria were recruited in the study which was conducted in Department of Otorhinolaryn-gology, Services Hospital, Lahore. Demographic information (name, age, gender, contact) was also obtained. Patients with clinical suspicion of impacted esophageal food bolus of age 20-50 years of either gender were included. Patients with known esophageal abnormalities like stricture, web or growth on either barium swallow or esophagogastroduodenoscopy, peptic ulcer and GERD were excluded. After taking informed written consent from each patient, single intravenous dose of buscopan 20mg was given to each patient. Each patient was followed by the researcher himself for 24 hours to assess the passage of food bolus.

Results: $20-50$ years was the age range in our study, with mean age being $34.10 \pm .63$ years. Majority of the patients $32(51.61 \%)$ were between 20 to 35 years of age. Out of 62 subjects, 37 (59.68\%) were male, 25 $(40.32 \%)$ were females with male to female ratio $1.5: 1$. In our study, Frequency of patients passing the impacted esophageal meat bolus by conservative management was found in $51(82.26 \%)$ patients.

Conclusion: This study concluded that there is a high frequency of patients passing the impacted esophageal meat bolus by conservative management.

Keywords: Esophagus, foreign body, conservative management.
\end{abstract}

\section{Introduction}

$\mathrm{T}$ he accidental impaction of a meatbolus in the esophagus is a common occurrence especially in an under-developed society. ${ }^{1}$ Afterear and nose, the esophagus is the third commonest site for foreign body impaction. ${ }^{2}$ up to eighty percent of impacted foreign bodies are held up just above the cricopharyngeus. ${ }^{3} 13$ episodes per 100,000 population of foreign body impaction are reported annually. ${ }^{4}$ In a study by Damghani $\mathrm{M}$ et $\mathrm{al}^{5}$, foreign bodies were detected in $77 \%$ of the esophagoscopies and in the

\footnotetext{
1. Ghulam Murtaza 2. Sarwat Hassan Syed

3. Damish Arsalan 4. Mohammad Qamar Nasir

5. Muhammad Awais Amin 6. Amir Ayub Awan

1. ENT, Fatima Memorial Hospital, Lahore

2,4. ENT-I, SIMS, Services Hospital, Lahore

3,5. ENT-II, SIMS, Services Hospital, Lahore

6. PGM, Lahore General Hospital Lahore

Correspondence:

Dr. Sarwat Hassan Syed

ENT-I, SIMS, Services Hospital, Lahore, sarwathsyed1278@gmail.com Submission Date: 10-05-2020

1st Revision Date: $\quad 19-09-2020$

Acceptance Date: $18-10-2020$
}

rest, inflammation, ulcer, and stricture formation was found. In another study, $77.8 \%$ were detected to have foreign bodies and in the remaining, soft tissue mass, bleeding, ulcer, edema, and abscess were reported. ${ }^{6}$

Higher incidence of foreign body ingestion occurs in pediatric population in between six months to six years of age while in adult population foreign body impactions more commonly occur among patients with known mental retardation, mental impairment caused by liquor and old age and psychiatric illnesses as reported by Lee et al. ${ }^{7}$ Usually, two types of foreign bodies are encountered, true foreign bodies (coins, buttons) and food related foreign bodies. Most Foreign body impactions are seen in children at the narrowest portion of the alimentary tract which is the cricopharyngeus that lies $15 \mathrm{~cm}$ from the upper central incisors. $^{1,8}$

Majority of impacted esophageal foreign bodies (80\%-90\%) pass spontaneously without any surgical intervention. ${ }^{9,10}$ However, approximately $10 \%-20 \%$ 
of cases of foreign body impactions require rigid or flexible endoscopic removal under general or local anesthesia, whereas, less than $1 \%$ will need open surgery for foreign body removal or to treat complications. ${ }^{10,11}$ American Society of Gastrointestinal Endoscopy (ASGE) guideline, for management of impacted foreign bodies, suggests immediate surgical intervention for patients who are having absolute dysphagia. ${ }^{12}$ If the patient is not in distress, they are observed for 24 hours, before opting for any invasive procedures. $^{13}$

Many pharmacological or non-pharmacological prokinetic agents have been used to dislodge the impacted food bolus with variable success. ${ }^{14,15}$ A survey conducted in UK showed that the majority of practitioners did not immediately proceed to rigid endoscopy; rather, they preferred antispasmodic drugs (83\%), the most common being hyoscine butylbromide (Buscopan) and diazepam, that usually allows the esophageal lumen to relax and facilitate the passage of the impacted bolus. ${ }^{16}$ Rate of complications increase in the hands of an inexperienced surgeonin managing unrecognized distal esophageal food bolus impactions. ${ }^{17}$ In a study, about $80 \%$ of cases, the impacted bolus may pass uneventfully through the gastrointestinal tract without endoscopic aid. $^{18}$

The purpose of this study was to determine the frequency of patients passing the impacted esophageal meat bolus by conservative method alone in local population. Previously the available literature on this is scarce, so our study will not only be a useful addition to the existing literature, but will also provide us with local statistics. As in majority of our setups, the patients with impacted meat bolus do not take the conservative trials, instead they are subjected to endoscopic removal or surgery, thus the results of our study will encourage the clinicians to develop a conservative approach in these particular patients, hence avoiding the invasive procedures in order to reduce the morbidity of the affected.

\section{Methods}

This was a descriptive, case series study conducted in the Department of Otorhinolaryngology Unit I, Services Hospital, Lahore, from 2nd September 2017 to 1 st March 2018. The calculated sample size was 62 cases with $95 \%$ confidence level, $10 \%$ margin of error and taking frequency of patients passing the impacted esophageal meat bolus by conservative method alone as $80.0 \% 5$ by using following formula.

$$
\begin{aligned}
\text { sample size }= & n \\
= & \left(Z_{-}(1-\alpha / 5 P(1\right. \\
& \left.-P))^{\wedge} 2\right) / d^{\wedge} 2
\end{aligned}
$$

Non-probability, Consecutive sampling was used.

Study cases between age of 20-50 years of both genders were selected according to the following criteria. Patients with clinical suspicion of impacted esophageal food bolus (Presence of any food bolus in esophagus on x-ray (radiolucent shadow) and with dysphagia (difficulty in swallowing), odynophagia (painful swallowing), anorexia (sense of vomiting) on history, andduration of symptoms of $\leq 24$ hours were included in the research.

Patients with known esophageal abnormalities like stricture, web or growth on either barium swallow or esophagogastroduodenoscopy (OGD), patients with history of peptic ulcer (assessed on history and medical record) and patients with history of gastroesophageal reflux disease (assessed on history and medical record) were excluded from the study.

After ethical approval and written informed consent from 62 patients presenting to ENT emergency of Services Hospital Lahore, who fulfilled the inclusion and exclusion criteria were recruited. A single intravenous dose of Buscopan 20mg was given to each patient. Each patient was followed by the researcher himself for 24 hours to assess the passage of food. The patients who continued to exhibit symptoms of impaction for more than 24 hours, were taken totheatre for endoscopic removal under GA. All patients who were able to eat and drink without any difficulty were discharged from the ward. This research data was recorded on a specially formulated proforma which consisted of two parts. Part 1 includes the patient's bio-data; part 2 consists the study variables.

\section{Statistical Analysis}

The data was processed using SPSS version 22 and analyzed. The results were presented as mean and standard deviation for quantitative variables i.e. age 
and duration of symptoms. Frequency and percentage were calculated for qualitative variables like gender, type of food bolus (meat/chicken/other including non-organic foreign bodies), site of impaction in esophagus (upper/middle/lower), previous history of food impaction (yes/no) and passage of impacted esophageal food bolus (yes/no).

Effect modifiers like age, gender, duration of symptoms, type of food bolus (meat/chicken/other), site of impaction in esophagus (upper/middle/lower) and previous history of food impaction (yes/no) were controlled through stratifications. Post-stratification chi square was applied to see their effects on outcome and $p$ value $\leq 0.05$ was considered as significant

\section{Results}

Age range in this study was from 20 to 50 years with mean age of $34.10 \pm 7.63$ years. Majority of the patients, 32 in number $(51.61 \%)$ were between 20 to 35 years.

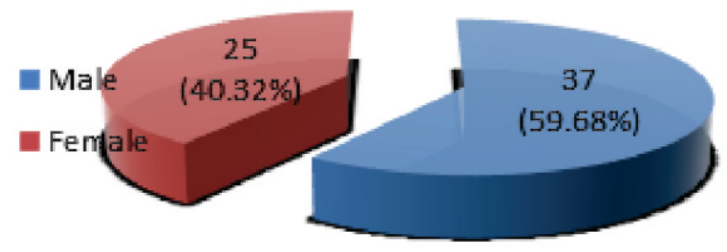

Figure I: Distribution of Patients According to Gender $(n=62)$.

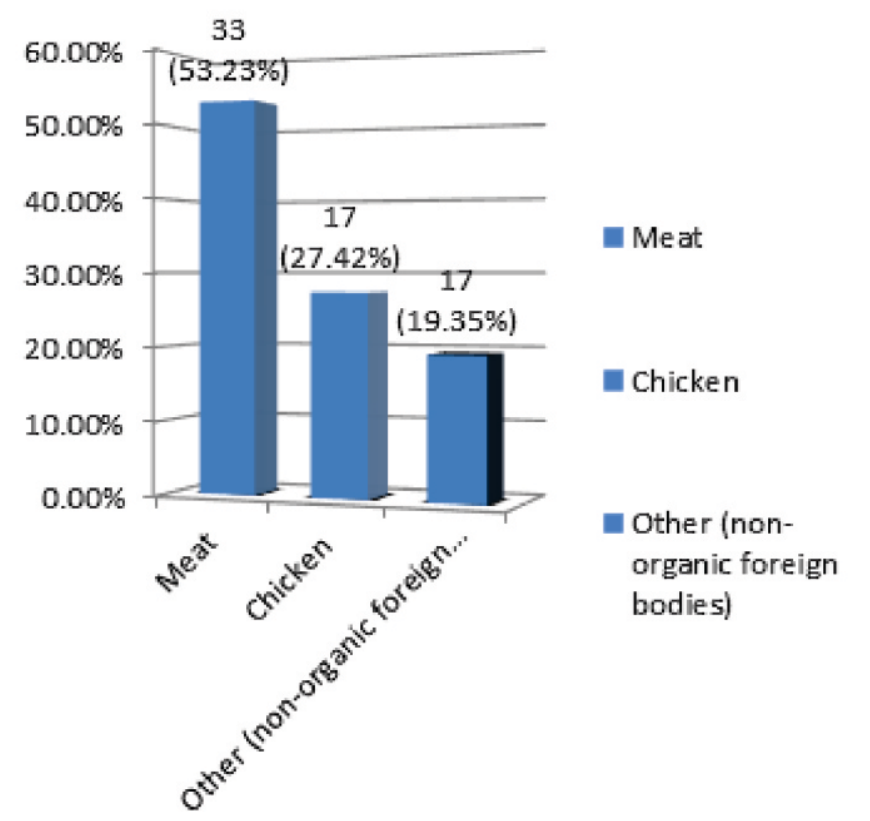

Figure II: Distribution of Patients According to the Type ofBolus $(n=62)$
Out of 62 patients, 37 (59.68\%) were male and 25 $(40.32 \%)$ were females with male to female ratio 1.5:1 as shown in Figure I. Mean duration of symptoms was $11.53 \pm 5.68$ hours. Distribution of patients according to the type of bolus as shown in Figure II.

In our study, Frequency of patients passing the impacted esophageal food bolus by conservative management was found in $51(82.26 \%)$ patients.

Table I \& II have shown the stratification of passage of impacted esophageal food bolus with respect to site of impaction and type of food bolus respectively.

Table 1: Stratification of Passage of Impacted esophageal Food Bolus with Respect to Site of Impaction in Esophagus.

\begin{tabular}{cccc}
\hline $\begin{array}{c}\text { Site of } \\
\text { impaction in } \\
\text { esophagus }\end{array}$ & \multicolumn{2}{c}{$\begin{array}{c}\text { passage of impacted } \\
\text { esophageal food bolus }\end{array}$} & \multirow{2}{*}{$\begin{array}{c}\text { p- } \\
\text { value }\end{array}$} \\
\cline { 2 - 3 } Upper & Yes & No & \\
Middle & 16 & 04 & \\
Lower & 18 & 05 & $\mathbf{0 . 6 0 7}$ \\
\hline
\end{tabular}

Table 2: Stratification of passage of impacted esophageal food bolus with respect to type of food bolus.

\begin{tabular}{lccc}
\hline Type of food bolus & \multicolumn{2}{c}{$\begin{array}{c}\text { Passage of impacted } \\
\text { esophageal food bolus }\end{array}$} & $\begin{array}{c}\text { p- } \\
\text { value }\end{array}$ \\
\cline { 2 - 3 } & Yes & No & \\
Meat & 28 & 05 & \\
Chicken & 15 & 02 & $\mathbf{0 . 2 7 7}$ \\
Other (non -organic & 08 & 04 & \\
foreign bodies) & & & \\
\hline
\end{tabular}

\section{Discussion}

Foreign body (FB) ingestion and esophageal food impactions are a common ENT emergency. In adults, FB ingestion occurs more commonly in those with psychiatric illnesses, alcohol intoxication, and incarcerated individuals in quest of secondary gain. ${ }^{19-22} \mathrm{~A}$ research conductedon 262 adult individuals with FB ingestion and impaction cases, $92 \%$ were deliberate, and $85 \%$ of patients had an underlyingmental health issue. Patients with underlying esophageal pathology often present with food bolus impaction. Known cases of congenital malformations are also at increased risk for FB impaction. Certain occupations like carpenters and tailors have higher chances of FB ingestion as they hold nails and pins in their mouth while working. ${ }^{23}$

We have conducted this study to determine the 
frequency of patients passing the impacted esophageal meat bolus by conservative method alone. Age range in this study was from 20 to 50 years with mean age of $34.10 \pm 7.63$ years. Majority of the patients 32 (51.61\%) were between 20 to 35 years of age. Out of 62 patients, $37(59.68 \%)$ were male and $25(40.32 \%)$ were females with male to female ratio 1.5:1. In our study, Frequency of patients passing the impacted esophageal meat bolus by conservative method was found in $51(82.26 \%)$ patients. A survey conducted among UK practitioners showed that the majority did not usually proceed immediately to rigid endoscopy for removal of meat bolus impaction; rather, they gave precedence to use of antispasmodic drugs $(83 \%)$, to try to induce spontaneous passage of the impacted meat bolus. ${ }^{16}$ In a study, in about $80 \%$ of cases, the ingested material passes uneventfully through theupper gastrointestinal tract. ${ }^{18}$

Success rate by conservative management alone was $68 \%$ better than the endoscopic approach in the study conducted by Basavaraj and Penumetcha. The literature search revealed one case study and two retrospective cohort studies comparing hyoscine butylbromide against no invasive treatment for esophageal meat bolus impaction. Another study has shown $82 \%$ success rate of conservative management using intravenous hyoscine. Hyoscine butylbromide is widely used in the management of esophageal meat bolus impaction. With the total of 74 subjects, the abovementioned studies concluded that there was no significant difference in disimpaction rates between those patients treated with hyoscine butylbromide and those who received no treatment. The lack of statistical supremacy to small sample sizes suggests further studies are needed. ${ }^{23-25}$ The evidence for hyoscine butylbromide as a treatment for esophageal soft food bolus obstruction is questioned in a publication from 2007. The evidence backing the use of hyoscine butylbromide appears to stem from a recommendation in a 1997 textbook that misquotes a 1991 study detailing 10 out of 16 patients which were given "antispasmodics", were spontaneously relieved of the obstruction. The study is questioned as a cohort of only 16 patients, which provides results of low statistical power and more relevantly, none of the 5 different "antispasmodic" drugs used in the study actually included hyoscine butylbromide.

A literature review showed that four retrospective cohort studies and one case report were acknowledged. These case studies stated disimpaction rates of $100 \%, 100 \%, 100 \%, 80 \%$ and $65 \%$, with prokinetics alone respectively. One patient suffered a mucosal tear to the oesophagus. The prokinetic agents vary in each study, the literature on the use of fizzy drinks in esophageal soft food bolus obstruction has a small patient number. Nevertheless, the results of the studies were positive, with $79 \%$ of cases experiencing disimpaction with a prokinetic agent alone or with barium meal for imaging. This combination therapy has not been formulated in a larger study. Larger trials are required to provide evidence in favor of their use. However, considering the fact that carbonated drinks are inexpensive, safe and apparently effective to some extent, their use in the management of esophageal soft food bolus disimpaction may be recommended. ${ }^{24}$

Other known pharmacological agents include glucagon, which reduces esophageal motility and relaxes the lower esophageal sphincter. However, it is not recommended to be effective in treating impacted esophageal coins in pediatric population, but it has been used in the managing esophageal soft food bolus obstruction. A comprehensive research revealed a randomized controlled trial showing no significant difference when comparing glucagon combined with diazepam against placebo for management of esophageal soft food bolus impaction. Two studies investigating 92 and 222 cases of esophageal soft food bolus obstruction were carried out. In the first study, all 92 patients were given glucagon and 30 were dis-impacted without endoscopy. 10 out of 106 patients were given glucagon and 20 out of 116 patients that were given no medication, dis-impacted without further interventionin the second study. ${ }^{21}$ The results suggest that administration of glucagon gives similar results in relieving esophageal soft food bolus obstruction as oppose to when no medication is given.

A large number of clinicians dealing with this emergency are not well versed with the protocols of treating patients suffering from food bolus impaction. This is the reason that small number of patients were taken to the OR within 12-hours observation, or they could not get any medical treatment. Such patients are exposed to unnecessary endoscopy and anesthesia related risks. Therefore, operating rooms and surgeons become overworked. 


\section{Conclusion}

This study concluded that there is a high frequency of patients passing the impacted esophageal meat bolus by conservative method alone. So, we recommend that conservative approach should be encouraged in these particular patients and avoid the invasive procedures in order to lessen the morbidity associated with rigid endoscopies.

\section{Author's Contributions}

DA: Data Collection

SHS: Introduction, literature review, Disscussion

GM,AAA: Article references

MQN: Statistical Analysis

MAA: Edited

\section{References}

1. Iqbal K, Khan MI, Muhammad. Esophageal foreign bodies: experience with rigid esophagoscopy at D.I. Khan. Gomal J Med Sci. 2012;10:194-7.

2. Akhtar M, Haq MI. Management of oesophageal foreign bodies. Professional Med J. 2008;12(3):30811.

3. Han S, Kayhan B, Dural K, Kocer B, Sakinci U. A new and safe technique for removing cervical esophageal foreign body. Turk J Gastroenterol. 2009; 16(2):108-10.

4. Ko HH, Enns R. Review of food bolus management. Can J Gastroenterol. 2008;22:805-7.

5. Damghani M, Halavati N, Motamedi N. Foreign body in the upper airway and oesophagus: a seven years study from Iran. J Pak Med Assoc. 2011; 61(9): 859-62.

6. Hashemi BS, Gandomi B, Hesamzade L. Evaluation of the Incidence and Complications of Foreign Body Ingestion in the Patients Referred to Shiraz Khalili Hospital. Armaghan Danesh 2005;32:42-8.

7. Lee TH, Kang YW, Kim HJ, Kim SM, Im EH, Huh $\mathrm{KC}$, et al. Foreign objects in korean prisoners. Korean Int Med J. 2007;22:275-78.

8. Muhammad R, Khan Z, Jamil A, Malik S, Haroon T, Khan F, et al. Frequency of esophageal foreign bodies and their site of impaction in patients presenting with foreign body aerodigestive tract. Eur Sci J. 2013; 9(21):152-60.

9. Dray X, Cattan P. Foreign bodies and caustic lesions. Best Pract Res Clin Gastroenterol. 2013;27:679-89.

10. Pfau PR. Removal and management of esophageal foreign bodies. Tech Gastrointest Endosc. 2014; 16: 32-9.

11. Birk $M$, Bauerfeind $P$, Deprez $P H$, Häfner $M$, Hartmann D, Hassan C, et al. Removal of foreign bodies in the upper gastrointestinal tract in adults: European Society of Gastrointestinal Endoscopy (ESGE) Clinical Guideline. Endoscopy. 2016; 48:1-8.

12. Sugawa C, Ono J, Taleb M. Endoscopic management of foreign bodies in the upper gastrointestinal tract: A review. World J Gastrointest Endosc. 2014;6:475-81.

13. Basavaraj S, Reddy CE, Dempster J, Reddy K, Kang M. Buscopan (hyoscine butylbromide) in the management of food bolus obstruction in oesophagus: randomised controlled trial. Internet J Otorhinolaryngol. 2015;18(1):1-5.

14. Kramer RE, Lerner DG, Lin T. Management of ingested foreign bodies in children: a clinical report of the NASPGHAN Endoscopy Committee. J Pediatr Gastroenterol Nutr. 2015;60:562-74.

15. Geraci G, Sciume C, Di Carlo G, Picciurro A, Modica G. Retrospective analysis of management of ingested foreign bodies and food impactions in emergency endoscopic setting in adults. BMC Emergency Med. 2016;16:42.

16. Price T, Jones SEM, Montgomery PQ. Is current UK management of oesophageal food bolus obstruction evidence based? An e-mail survey and literature review. European ArchivES Otorhinolaryngol. 2007; 264(4):329-35.

17. Khayyat YM. Pharmacological management of esophageal food bolus impaction. Emerg Med Int. 2013;2013:924015.

18. Ambe P, Weber SA, Schauer M. Swallowed foreign bodies in adults. Dtsch Arztebl Int. 2012; 109: 86975.

19. Webb WA. Management of foreign bodies of the upper gastrointestinal tract: Update. Gastrointest Endosc 1995;41:39-51.

20. Cheng W, Tam PK. Foreign-body ingestion in children: Experience with 1,265 cases. J Pediatr Surg 1999;34:1472-6.

21. Hachimi-Idrissi S, Corne L, Vandenplas Y. Management of ingested foreign bodies in childhood: Our experience and review of the literature. Eur $\mathbf{J}$ Emerg Med 1998;5:319-23.

22. Panieri E, Bass DH. The management of ingested foreign bodies in children - A review of 663 cases. Eur J Emerg Med 1995;2:83-7.

23. Palta R, Sahota A, Bemarki A, Salama P, Simpson N, Laine L. Foreign-body ingestion: Characteristics and outcomes in a lower socioeconomic population with predominantly intentional ingestion. Gastrointest Endosc 2009; 69 (3 Pt 1):426-33.

24. Weiland ST, Schurr MJ. Conservative management of ingested foreign bodies. J Gastrointest Surg 2002;6:496-500.

25. Blaho KE, Merigian KS, Winbery SL, Park LJ, Cockrell M. Foreign body ingestions in the emergency department: Case reports and review of treatment. J Emerg Med 1998;16:21-6. 